\title{
Ansiedad y procrastinación en estudiantes universitarios
}

\author{
Anxiety and procrastination in university students
}

Recepción del artículo: 25-11-18| Aceptación del artículo: 08-12-18

Marquina-Luján, Román Jesús* roman.marquina@usil.pe ORCID: 0000-0002-5542-7234

Horna Calderón, Víctor Eduardo* victor.hornac@usil.pe ORCID: 0000-0002-3071-5491

Huaire Inacio, Edson Jorge* edson.huaire@usil.pe ORCID: 0000-0003-2925-6993

Universidad San Ignacio de Loyola*

Para referenciar este artículo:

Marquina Lujan, R. J., Horna Calderón, V. E. y Huaire Inacio, E. J. (2018). Ansiedad y procrastinación en estudiantes universitarios. Revista ConCiencia EPG, 3(2), 89-97. doi: 10.32654/CONCIEN-

CIAEPG.3-2.6

\section{Resumen}

a presente investigación tuvo como objetivo determinar la relación existente entre procrastinación académica y ansiedad en un grupo de estudiantes universitarios ingresantes en una universidad privada de Lima Metropolitana. Se empleó un diseño no experimental de tipo correlacional. Los instrumentos de medida empleados fueron la escala de procrastinación académica (EPA) adaptada por Álvarez (2010) y la escala de autoevaluación de la ansiedad de Zung adaptada por Astocondor (2001). El análisis de ellos, dieron un valor de alfa de cronbach de 0.79 y 0.81 respectivamente, indicándonos su nivel de fiabilidad. Los resultados evidencian que las variables de procrastinación académica y ansiedad presentaban una correlación negativa, no obstante, los puntajes de ansiedad y procrastinación académica son moderados. Se concluye por tanto que, los estudiantes que procrastinan no presentan ansiedad debido posiblemente a que la actitud de procrastinar no afecta su desempeño y tampoco es un factor psicológico que lleva a una conducta de ansiedad.

Palabras clave: Procrastinación académica; ansiedad; síntomas somáticos A; Síntomas cognoscitivos A; síntomas somáticos B; Síntomas cognoscitivos B 


\begin{abstract}
The objective of the present investigation was to enter a private university in Metropolitan Lima, whose ages fluctuated between 18 and 23 years old, selected by means of an intentional non-probabilistic sampling, selected through an intentional non-probabilistic sampling. A nonexperimental correlational type design was used given that its purpose was to establish the association between two or more study variables in a particular context, in the same group of subjects (Hernández, Fernández \& Baptista, 2014). The instruments of measurement were the Scale
\end{abstract}

\section{Introducción}

1 termino de ansiedad ocupa un
lugar privilegiado en los estudios
psicológicos y clínicos, asociado también a otros constructos como estrés, angustia, etc. Según Agudelo, Casadiegos y Sánchez (2008), la ansiedad es uno de los trastornos psicológicos más registrados en los centros de salud y en la población en general. Marks (1986) sostiene que, es una combinación de manifestaciones físicas y psicológicas, expresadas ya sea como una crisis, o bien como un estado de ánimo, por lo general, este estado se presenta en función a la interpretación de peligro que percibe la persona, lo cual no puede ser atribuidas a situaciones reales, sino, más bien están relacionadas con la preocupación ante futuros peligros que no pueden ser definidos o previstos de manera concreta. of Academic Procrastination (EPA) adapted by Alvarez (2010) and the Self-Assessment Scale of Zung's Anxiety adapted by Astocondor (2010). In the analysis of results, it was found that the variables of anxiety and academic procrastination had a positive correlation, although the scores of anxiety and academic procrastination are mostly moderate.

Key words: Academic Procrastination; Anxiety; somatic symptoms A; Cognitive symptoms A; somatic symptoms B; Cognitive symptoms B

De acuerdo con los estudios de Martínez (2014) en el ámbito universitario existe una presencia elevada de ansiedad, los cuales pueden tener impacto negativo en la salud mental y favorecer la aparición de ansiedad patológica (Bhave y Nagpal, 2005); los niveles excesivos de ansiedad, en la mayoría de los casos también, puede tener efectos insidiosos en la trayectoria del estudiante, como el descenso de su rendimiento académico, deterioro de sus relaciones interpersonales y de su salud. Por el contrario, un nivel equilibrado de ansiedad ayuda por ejemplo, a afrontar los retos que se presentan en la vida universitaria, debido a que actúa como un movilizante que permite buscar estrategias de salida de la situación de ansiedad y se compensa con el bienestar psicológico. 
Por otro lado, la procrastinación, es un comportamiento que está frecuentemente asociado al rechazo a las exigencias e imposiciones y caracterizado por una falta de organización y responsabilidad, sea en el ámbito académico, familiar o laboral (Pardo, Perilla \& Salinas, 2014). El concepto clásico de procrastinación hacía referencia a la pérdida o evitación voluntaria e innecesaria de tiempo y el incumplimiento de tareas, este comportamiento se comprendió como una característica negativa del ser humano. Hacía referencia, entonces, a la postergación voluntaria de tareas que deben ser entregadas en un determinado tiempo (Ferrari, Driscoll \& Diaz 2007; Quant \& Sánchez, 2012).

En algunos estudios, se ensaya una clasificación de las personas que procrastinan, como el realizado por Takás (citado en Angarita, 2012) quien considera siete tipos: el primero llamado el perfeccionista, cuyas tareas o responsabilidades resultan aplazables debido a que no alcanzan sus estándares personales, el segundo llamado soñador, se caracteriza más bien por divagar y ser poco realista, el tercero es el preocupado, quien procrastina en los momentos que siente que las cosas están fuera de su control, un cuarto tipo es el generador de crisis, quien disfruta de las interacciones que se asocian con el aplazamiento. El desafiante es el quinto tipo de procrastinador, quien utiliza la agresión como excusa de control a terceros, un sexto tipo es el ocupado, que nunca tiene tiempo y, por último, el relajado caracterizado por un tipo de persona que evita situaciones y actividades que le generan algún tipo de estrés y compromiso. Asimismo, en esta línea, encontramos a Hsin y Na, (citado por Quant y Sánchez, 2012) quienes agrupan a los procrastinadores en dos tipos: pasivos y activos. Los primeros que describen a quienes no realizan por completo una tarea, mientras que los segundos necesitan estar bajo presión para cumplir las tareas encomendadas, y cuyas decisiones son tomadas de forma apresurada.

Respecto a la relación que existe entre estos constructos, se puede afirmar que la conducta procrastinadora con frecuencia dificulta y perjudica el éxito académico debido a que los estudiantes, por lo general, reemplazan las tareas académicas por otras actividades como dormir, jugar, ver televisión etc., (Binder, 2000) lo cual constituye una importante fuente de malestar psicológico que puede llegar a perjudicar la salud de los estudiantes (Natividad, 2014), sobre todo a elevar el nivel de ansiedad debido a la persistente demora en las tareas académicas.

Sin embargo, es necesario confirmar resultados en el grado de relación que existe entre estos constructos, dado que hay diferentes resultados a favor de una relación positiva (Domínguez, 2017; Gil, 2018; Pardo, Perilla y Salinas, 2014; y una relación débil (Mamani, 2017). Por ello, es importante seguir profundizando en el estudio, para poder clarificar de manera científica la asociación de las variables. 


\section{Método}

Diseño: el diseño del estudio es correlacional de tipo transversal dado que su propósito fue establecer la asociación entre las variables, procrastinacion académica y ansiedad, en el mismo grupo de sujetos.

Participantes: fueron considerados un total de 100 estudiantes universitarios del primer año de estudios generales de una universidad particular, cuyas edades fluctuaban entre los 18 y 23 años, 52 de los cuales fueron de sexo femenino y 48 del masculino. La selección de los participantes fue favorecida por la disponibilidad de acceso a la muestra.

Instrumentos: los instrumentos utilizados para esta investigación fueron en primer lugar, la Escala de Procrastinación Académica (EPA) desarrollada por Busko en el año1998 y adaptada por Álvarez en el año 2010, consta de 16 ítems de elección múltiple (1) siempre me ocurre, (2) casi siempre o alguna vez me ocurre, (3) a veces me ocurre, (4) pocas veces me ocurre y (5) nunca me ocurre. Lo cual permite evaluar la tendencia hacia la procrastinación académica. El alfa de cronbach obtenido fue de 0.80 lo cual nos señala el nivel de fiabilidad que presenta el instrumento. El análisis realizado por este autor permite confirmar que en este instrumento existe un solo factor que explica el $23,89 \%$ de la varianza total.

Como segundo instrumento, la Escala de Autoevaluación de la ansiedad de ZUNG (E.A.A). Desarrollada por Zung en el año 1965 y adaptada por Astocondor en el año 2010, comprende de 20 informes o relatos, cada uno referido a manifestaciones características de ansiedad, como síntomas o signos como expresión de trastornos emocionales. Sus preguntas generalmente indican el deseo de cooperación con el profesional de la salud (médico, psicólogo) y deben ser respondidas de tal manera que induzcan a apersona a dar las respuestas más acordes a su realidad personal, sus respuestas son de tipo Likert (1) nunca casi nunca, (2) a veces, (3) con bastante frecuencia, (4) siempre y (5) casi siempre. Cabe señalar que se pudo obtener el alfa de Cronbach, el cual dio un valor de 0.81 lo cual indica que el nivel de fiabilidad es adecuado. La escala está conformada por cuatro dimensiones síntomas somáticos A “dolores, mareos, temblor, taquicardia y debilidad, desmayo" $(7,11,18,8,6,10,12,15)$ Síntomas cognoscitivos A "nerviosismo, irritabilidad, pesadillas" (2, 1, 20,3) síntomas somáticos B "poliuria, adormecimiento dedos, desbaratarse" $(16,4,14,17)$ Síntomas cognoscitivos B "inquietud, presentimientos negativos y disnea" $(5,9,13,19)$.

Procedimiento: para la presente investigación previamente se presentó un oficio a la Dirección Académica de la universidad, a fin de obtener la autorización para el ingreso a las aulas, donde se encontraban los estudiantes del primer año de estudios en los turnos de la mañana y tarde. Una vez conseguido el permiso, se asistió a las aulas y se les informó a los estudiantes sobre el propósito de la investigación y se les solicitó su colaboración voluntaria, lo cual fue aceptado mediante la firma de una hoja de consentimiento in- 
formado. A partir de ello, procedieron a la resolución colectiva de los instrumentos, realizada en una sola sesión que duró aproximadamente $20 \mathrm{mi}$ nutos para ambas pruebas. Luego de haber reco-

\section{Resultados}

Siendo el objetivo de la presente investigación, establecer una correlación entre las variables procrastinación y ansiedad, en estudiantes

Tabla 1 Exploración de la normalidad de las variables procrastinación académica y ansiedad a través de las pruebas de Kolmogorov-Smirnov

\begin{tabular}{llcc}
\hline \multicolumn{1}{c}{$\mathbf{N}^{\circ}$} & $\begin{array}{c}\text { Procrastinación } \\
\text { académica }\end{array}$ & Ansiedad \\
\hline Parámetros normales & Media & 100 & 100 \\
& Desviación típica & 48.38 & 53.79 \\
& Absoluta & 8.307 & 5.370 \\
Diferencias extremas & Positiva & 0,073 & 0,70 \\
Z de Kolmogorov-Smirnov & Negativa & 0,73 & 0,070 \\
Sig. Asintót (bilateral) & & 0,727 & 0,703 \\
& & 0,666 & 0,706 \\
\hline
\end{tabular}

Se partió con la evaluación de la prueba de norMediante una diferenciación de medias, malidad de Kolmogorov - Smirnov, cuyos resultados permitieron concluir que la prueba de procrastinación académica como la de ansiedad presentan normalidad estadística de acuerdo a sus valores $p=, 666$ y, 706 respectivamente, por consiguiente, se puede dar inicio a un análisis paramétrico de las variables. gido los datos, éstos fueron ingresados al programa estadístico SPSS versión 23, en donde se hicieron los respectivos análisis descriptivos e inferenciales.

universitarios se pasa a detallar los resultados encontrados. se pudo determinar que la distribución de la muestra es homogénea en ambas variables, lo cual se expresa en los puntajes de la media, así como de la desviación estándar. Sin embargo, se observó que existe una mayor desviación en los puntajes de procrastinación del género femenino que en los del género masculino; por otro lado, en referencia a la variable ansiedad, se puede ver que la diferencia entre sus desviaciones estándar es mucho más pequeña. 
Tabla 2

Descripción de la procrastinación académica y ansiedad en estudiantes universitarios

\begin{tabular}{ccccccccc}
\hline & \multicolumn{3}{c}{ Procrastinación académica } & \multicolumn{3}{c}{ Ansiedad } \\
\cline { 2 - 9 } & \multicolumn{2}{c}{ Varones } & \multicolumn{2}{c}{ Mujeres } & \multicolumn{2}{c}{ Varones } & \multicolumn{2}{c}{ Mujeres } \\
\cline { 2 - 9 } & $\mathrm{f}$ & $\%$ & $\mathrm{f}$ & $\%$ & $\mathrm{f}$ & $\%$ & $\mathrm{f}$ & $\%$ \\
\hline Bajo & 13 & $27.08 \%$ & 26 & $50 \%$ & 23 & $47.92 \%$ & 19 & $36.54 \%$ \\
Medio & 20 & $41.67 \%$ & 22 & $42.31 \%$ & 19 & $39.58 \%$ & 19 & $36.54 \%$ \\
Alto & 15 & $31.25 \%$ & 4 & $7.69 \%$ & 6 & $12.5 \%$ & 14 & $26.92 \%$ \\
TOTAL & $\mathbf{4 8}$ & $\mathbf{1 0 0 \%}$ & $\mathbf{5 2}$ & $\mathbf{1 0 0 \%}$ & $\mathbf{4 8}$ & $\mathbf{1 0 0 \%}$ & $\mathbf{5 2}$ & $\mathbf{1 0 0}$
\end{tabular}

A nivel descriptivo podemos apreciar respecto a la procrastinación que existe un predominio en un nivel medio alto respecto a la muestra evaluada y esta predominancia alta se observa más en varones que en las mujeres. En cuanto a la ansiedad

Tabla 3

Correlación entre las variables procrastinación académica y ansiedad

\begin{tabular}{llcc}
\hline & & Procrastinación & Ansiedad \\
\hline Procrastinación & Correlación de Pearson & 1 &,- 072 \\
& Sig (bilateral) & &, 475 \\
Ansiedad & Correlación de Pearson &,- 072 & 1 \\
& Sig (bilateral) &, 475 & \\
\hline
\end{tabular}

Se puede apreciar la correlación entre los puntajes de procrastinación académica y ansiedad es $(r=-, 072)$; esto nos quiere decir que, si percibida se halló que con mayor frecuencia esta se ubica en los niveles medios y bajos; sin embargo, hay que señalar que son las mujeres quienes obtienen mayor puntuación en el nivel alto.

\section{Discusión}

Según lo obtenido mediante este estudio, las variables ansiedad y procrastinación académica presentan una correlación significativa- los niveles de procrastinación académica aumentan, los niveles de ansiedad disminuyen, dado que sabemos que a mayor exageración siempre hay una consecuencia. 
equilibrados resulta beneficiosa, pues ayuda, por ejemplo, a afrontar los retos que se presentan en la vida universitaria.

Los resultados encontrados en este estudio coinciden con los reportes de Pardo, et al. (2014), quien encontró relación significativa baja entre procrastinación y ansiedad; sin embargo, difieren en cuanto a la dirección de la relación, ya que ellos refieren que a mayor procrastinación resultaba también, mayor ansiedad y a menor procrastinación menor ansiedad. Igualmente Sánchez (2010), afirma que la ansiedad y la procrastinación académica se correlacionan de forma significativa, tomando en cuenta que el comportamiento de procrastinación académica no solo se entiende como una falta de competencia en la gestión del tiempo dedicado al estudio y a concluir la labor de forma eficaz, sino que, la ansiedad se consideraría como una causa relevante sumada a la percepción de un estresor, como una exigencia académica, lo que genera en los sujetos respuestas emocionales que se caracterizan como un malestar.

Por lo tanto, existe una tendencia a evitar la realización de las actividades académicas como una forma de evadir dichas situaciones asociadas a consecuencias punitivas y ansiógenas que finalmente se traducen en emociones negativas o inadecuadas. Yarlequé et al. (2016) reportaron que, en los procrastinadores tienden a presentarse menos bienestar psicológico, aumentando sus niveles de estrés, así como pocos recursos para manejar la situación. En cuanto a los resultados del estudio que presentamos, podríamos inferir a que ya los niveles de ansiedad en la muestra son altos a nivel descriptivo más del $50 \%$ de los encuestados presentan niveles de ansiedad medio y alto, y los niveles de procrastinación en su mayoría están en el nivel medio. Estos se deberían probablemente a que la ansiedad que presentan los estudiantes se deba a otros factores y no necesariamente a la conducta de postergación lo cual se presenta entre el medio y bajo, es decir, dado que no procrastinan su ansiedad no se debe a ese comportamiento, sino, como ya se ha explicado se deba a otros factores.

Con respecto a la variable sociodemográfica género, se pudo establecer la existencia de diferencia en cuanto a la procrastinación y en la ansiedad, se pudo comprobar que los varones procrastinan más que las mujeres, aunque dicha diferencia no es demasiado firme entre ambos sexos. Coincidentemente el estudio de Chan (2011) encontró que existen diferencias entre hombres y mujeres en cuanto al comportamiento de procrastinación académica, sin embargo, las diferencias no son distantes, pues los hombres tienden a procrastinar más que las mujeres, y las mujeres estarían potencialmente en camino a la procrastinación. Por otro lado, se halló que la ansiedad percibida en los estudiantes universitarios se presenta con mayor frecuencia en las mujeres.

Consideramos que la procrastinación académica es un problema complejo que no obedece a un solo factor en especial, sino que existen un conjunto de situaciones que pueden explicar su presencia. Algunos estudios precederos, ya lo ha- 
bían señalado, como es el caso de Howell y Watson (citado por Angarita, 2012) quienes advierten que los comportamientos de dilación o procrastinación, no sólo implican factores intrínsecos sino también se relacionan con variables ambientales externas tales como el ruido, sobrecarga de tareas académicas o laborales, lo que a su vez al convertir la procrastinación en un hábito (presencia cotidiana) provocan los estudiantes que la manifiestan, problemas en su ámbito personal, académico, laboral, social y hasta familiar (Marquina, Gómez-Vargas, Salas-Herrera, Santibañez-Gihua, \& Rumiche-Prieto 2016).

\section{Referencias}

Agudelo, D. M., Casadiegos, C. P. \& Sánchez, D. L. (2008). Características de ansiedad y depresión en estudiantes universitarios. International Journal of Psychological Research, 1(1), 34-39.

Angarita, L. (2012). Aproximación a un concepto actualizado de la procrastinación. Revista iberoamericana de psicología: ciencia y tecnología 2, 85-94.

Álvarez, Ó. (2010). Procrastinación general y académica en una muestra de estudiantes de secundaria de Lima Metropolitana. Persona 159-177.

Astocondor, L. (2010). Estudio exploratorio sobre ansiedad y depresión en un grupo de
Por otro lado, la Procrastinación como fenómeno, no es patrimonio exclusivo del ámbito académico, ya que puede presentarse en diferentes escenarios donde exista una tarea o responsabilidad que cumplir. Los estudios de Steel (citado por Quant y Sánchez, 2012) aseveran que la procrastinación se presenta en varios escenarios, como el académico, laboral, político y bancario, donde la finalidad es la misma, salvar de forma voluntaria la consecución o elaboración de una tarea u obligación.

pobladores de comunidades nativas aguarunas (Tesis de licenciatura). Universidad de San Martín de Porres, Lima, Perú

Binder, K. (2000). The effects of an academic procrastination treatment on student procrastination and subjective wellbeing. Carleton University Ottawa: Ontario,

Chan, L. (2011). Procrastinación académica como predictor en el rendimiento académico en jóvenes de educación superior. Temas de Psicología, 7(1), 53-62.

Domínguez, S. (2017). Procrastinación académica, afrontamiento de la ansiedad preexa- 
men y rendimiento académico en estudiantes de psicología: análisis preliminar. Cultura, 31, 181-193. Doi: https://doi.org/10.24265/cultura.2017.v31.10.

Ferrari, J., Driscoll, M. \& Diaz, J. (2007). Examinn the Self of Chornic Procrastinators: Actual, Ought, and Undesired Attributes, 5(2), 115-123

Gil, L. (2018). Procrastinación académica y ansiedad en estudiantes de Ciencias de la Salud de una Universidad de Lima Norte. Revista Casus, 3(2), 89-96.

Mamani, S.E. (2017). Relación entre la procrastinación académica y ansiedad-rasgo en estudiantes universitarios pertenecientes al primer año de estudios de una Universidad Privada de Lima Metropolitana (Tesis de Licenciatura). Universidad Peruana Cayetano Heredia, Lima, Perú.

Marks, I. (1986). Tratamiento de neurosis. Barcelona: Martínez Roca

Marquina-Luján, R. J., Gómez-Vargas, L., Salas-Herrera, C., Santibañez-Gihua, S. \& Rumiche-Prieto, R. (2016). Procrastinación en alumnos universitarios de Lima
Metropolitana. Revista Peruana de Obstetricia y Enfermería, 12(1). Recuperado de http://www.aulavirtualusmp.pe/ojs/index.php/rpoe/article/view/747

Martínez, V. (2014). Ansiedad en estudiantes universitarios: estudio de una muestra de alumnos de la Facultad de Educación”, en ENSAYOS, Revista de la Facultad de Educación de Albacete, № 29-2. Recuperado de http://www.revista.uclm.es/index.php/ensayos.

Pardo, D., Perilla, L. \& Salinas, C. (2014). Relación entre procrastinación académica y ansiedad-rasgo en estudiantes de psicología. Cuadernos Hispanos de Psicología. 14(1) 31-44.

Quant, M. \& Sánchez, A. (2012). Procrastinación, procrastinación académica, concepto implicaciones. Revista vanguardia psicológica, 3, 44-55.

Yarlequé, L. (2016). Procrastinación, estrés y bienestar psicológico en estudiantes de educación superior de Lima y Junin. Horizonte de la Ciencia, 6(10), 173-184. Recuperado de http://www.uncp.edu.pe/revistas/index.php/horizontedelaciencia/article/download/213/20 9 